\title{
MODELOS DE ESCOLARIZACIÓN: TRAYECTORIA HISTÓRICA DE LA EDUCACIÓN ESPECIAL
}

\author{
Teresa González Pérez*
}

\section{RESUMEN}

El objetivo de este trabajo es estudiar el itinerario legislativo del sistema educativo español en orden a las discapacidades psíquicas y físicas. Desde la Ley Moyano a la Ley General de Educación la segregación escolar fue una realidad en la escuela española. El currículum y la enseñanza diferenciada han marchado estrechamente unidas a la trayectoria de la educación especial en España, segregando a los alumnos y alumnas de las aulas ordinarias. La educación con la inercia que le caracteriza, ha ido reproduciendo y legitimando situaciones establecidas, y con ello, desigualdades bajo un marco normativo que garantizaba la igualdad de oportunidades. En la actualidad la Ley de Ordenación General del Sistema Educativo (LOGSE) pretende hacer realidad "la escuela para todos" que incluya a todos sus miembros independientemente de sus peculiaridades personales. La escuela inclusiva debe dar respuesta a la diversidad de realidades del alumnado, como lugar abierto y flexible para aquellos que tradicionalmente han sido descalificados por sus limitaciones físicas y/o psíquicas.

Palabras clave: Historia. Educación. Marginación. Segregación. Discapacidad. Legislación. Diversidad.

\begin{abstract}
The aim of this paper is to study the legal itinerary of the Spanish educative system on behalf of students with mental and physical disabilities. Since

*Catedrática da Escola Universitária, Doutora em História pela Universidade de La Laguna e Professora da Faculdade de Educação da Universidade de La Laguna (Espanha).E-mail: teregonz@ull.es
\end{abstract}


the Moyano Law to the General Law on Education school segregation was a reality within the Spanish schools. Curricula have always gone hand in hand with the path of Special Education in Spain, segregating male and female students from the regular lessons. Education has reproduced and sanctioned inequalities within the legal framework that guaranteed equal opportunities. Nowadays, the LOGSE wants to establish a "school for everybody" policy that includes all its members no matter their personal peculiarities. The inclusive school must find a solution to students' diversity.

Keywords: History. Education. Marginalization. Segregation. Disability. Legislation. Diversity.

\section{Introducción}

En España la incorporación a la escolaridad de los niños y niñas con discapacidad no ha estado exenta de dificultades. Invisibles y marginados por la sociedad su trayectoria educativa ha sido lenta y sinuosa. El itinerario legislativo del sistema educativo español ha mantenido graves situaciones de desigualdad, no sólo con respecto al género sino también en orden a las capacidades psíquicas y /o físicas. El reconocimiento político a este colectivo se ha plasmado en el desarrollo de una normativa y su tratamiento educativo ha venido marcado por los principales hitos legislativos. La primera ley de instrucción pública española, promulgada en 1857 y conocida como ley Moyano, dispuso la creación de una escuela para ciegos y sordos en cada distrito universitario. A pesar de la diversa gama englobada en la educación especial, en un principio sólo se atendía a los sensoriales. Aquella infancia que tenía otra discapacidad quedaba fuera del alcance del marco normativo, y al margen de la deficiencia sensorial, no se atendió la escolarización de la infancia con otro tipo de discapacidad hasta 1970. De manera que buena parte de los niños y niñas con discapacidad no recibían instrucción. La mentalidad social imperante condicionaba la proyección educativa y la atención que debía recibir la infancia discapacitada. Un arduo camino lleno de obstáculos y estereotipos que condenaba a los disminuidos a la ignorancia y a la desatención. Además de que escapaban a la acción educativa, a todos y todas se les 
refería con términos despectivos. Empleaban un amplio repertorio de una nomenclatura discriminatoria, usando sinónimos que iban desde la denominación de retrasados, anormales, subnormales, débiles mentales o tullidos a llamarlos también idiotas, imbéciles, bobos o tontos. En suma un desprecio que en buena parte de los casos se proyectaba también en el ámbito familiar con la ocultación de los afectados, manteniendo a niños, jóvenes o adultos encerrados en las casas y alejados de la vida social. Una visión teñida por los estereotipos sociales y amparada en una conducta contaminada por las creencias y construcciones culturales de lo que se consideraba "normal" o saludable.

A lo largo del siglo XX la educación española se fue transformando al mismo tiempo que evolucionaba la sociedad, pero especialmente a partir del último tercio de la centuria los cambios fueron notorios. Las importantes modificaciones que se gestaron en el tramo final de la dictadura franquista tuvieron su reflejo e influencia en las estructuras y formas de entender la educación. La renovación pedagógica y los avances de la psicología educativa fueron elementos claves en la atención de la infancia. Por primera vez se prestaba atención a la diversidad de alumnado y a su escolarización con el objetivo de integración. Ciertamente los cambios operados junto a la recepción de innovaciones psicoeducativas y nuevos planteamientos pedagógicos contribuyeron de forma decisiva al desarrollo de la Educación Especial. Las propuestas educativas respondían a sus necesidades y pretendían dar respuesta a su problemática. En 1970 con la Ley General de Educación (LGE) se adoptó el término Educación Especial. Se contemplaba como una modalidad educativa específica con un currículo propio. Pero la Ley preveía la creación y puesta en funcionamiento de aulas de Educación Especial para niños con deficiencias en los colegios ordinarios. Con la aprobación de la LOGSE se produce un cambio fundamental en la concepción de la Educación Especial, formando parte del régimen general de educación y con atención en todos los niveles. De forma progresiva la educación fue incluyendo a aquellos niños que tenían algún tipo de limitación. En el nuevo milenio la integración ha quedado desfasada y los objetivos de la educación se concretan en la inclusión de todo el alumnado en aulas y centros educativos ordinarios. Un enfoque que reconoce las diferencias y elimina las barreras para conseguir una sociedad 
más igualitaria en un mundo en transformación. El objetivo de este trabajo es realizar la reconstrucción histórica del proceso seguido en el tratamiento educativo a sujetos con necesidades educativas especiales.

\section{Antecedentes educativos y legislativos}

Las primeras referencias conocidas en España sobre atención educativa especial datan del siglo XVI con la educación de sordos. Contamos con los precedentes de los educadores Pedro Ponce de León (1507?-1584) y Juan Pablo Bonet (1573-1633)1 (GASCÓN; STORCH, 2004; VICENTE; VICENTE, 2001). Las experiencias del fraile benedictino Pedro Ponce de León se remontan a $1550^{2}$; fue el iniciador de la enseñanza para sordos y creador del método oral (GASCÓN; STORCH, 2006). Transcurrieron varios siglos sin que se registraran avances y propuestas para la educación especial, si exceptuamos las aportaciones de Lorenzo Hervás y Panduro (1735-1809) y y la creación del Instituto de Sordomudos en 1786 (GASCÓN; STORCH, 2004). Hasta el siglo XIX prácticamente no hubo novedades. En 1800 el ayuntamiento de Barcelona creó una escuela para sordomudos y en 1802 funcionaba en Madrid la Real Escuela de Sordomudos impulsada por la Real Sociedad Económica de Amigos del País. Igualmente esta institución apoyó la creación de una escuela para ciegos ${ }^{4}$, que en 1836 figuraba adscrita al colegio de sordomudos. En 1852, por Real Decreto de

\footnotetext{
${ }^{1}$ Manuel Ramírez de Carrión (1579-1652) educador de varios sordos de la nobleza castellano, su método oral tuvo proyección cuando Juan Pablo Bonet publicó el libro "Reducción de las letras y arte para enseñar a hablar a los alumnos" en 1620.

${ }^{2}$ Ponce de León destacó por la atención de niños sordomudos aunque no formó escuela, superando antiguos prejuicios educó en el monasterio de Oña (Burgos) a doce niños y jóvenes sordomudos.

${ }^{3}$ El abate Lorenzo Hervás y Panduro (1735-1809) fue uno de los escritores más prolijos de la ilustración española. Destacó como pedagogo, significándose especialmente en la defensa del derecho a la educación de los sordomudos.

${ }^{4}$ Real Orden del 2 de abril y de 19 de diciembre de 1835, por la que el gobierno regula su creación a instancias de la Real Sociedad Económica de Amigos del País de Madrid. En 1836 estableció las bases para su funcionamiento y en 1841 le concedió la subvención de 28 mil reales.
} 
16 de Enero, el Real Colegio de Ciegos y Sordomudos pasó a depender del Ministerio de Fomento, una vez reconocido como establecimiento de instrucción pública (VICENTE; VICENTE, 2001). En 1863 se declaró de ámbito nacional al Colegio Nacional de Ciegos de Madrid y se manifestó por primera vez en España la necesidad de implantar "el Braille como sistema de lecto-escritura para ciegos" (GASCÓN; STORCH, 2004, p. 369). El Sistema Braille fue introducido en España en el año 1840 por Jaime Bruno Berenguer, profesor de la Escuela Municipal de Ciegos de Barcelona, con el deseo de que la educación de los ciegos siguiera los niveles y procedimientos de la educación de los videntes, pero la respuesta fue muy desigual. Transcurrió un amplio periodo de tiempo para que se generalizara el uso de esta metodología y no fue declarado como método oficial para la lectura y la escritura de los ciegos españoles hasta 1918 (ESPAÑA, 2002).

Sin embargo, no disponemos de antecedentes legislativos hasta mediados del siglo XIX. Es preciso remontarse hasta 1857 con la promulgación de la Ley de Instrucción Pública para que la normativa contemplara la educación de deficientes sensoriales en centros especiales. Dicho marco legislativo disponía la creación de una escuela para ciegos y sordos en cada distrito universitario, pero el resto de las deficiencias quedaban al margen del sistema educativo. Además especificaba de que se atendieran "en la medida de lo posible" en las escuelas públicas la enseñanza de los que tipificaban como "aquellos desgraciados"5. En 1875 se creó un Colegio Especial en Madrid a instancias de Carlos Nebreda y Pedro Espinosa. En aquel contexto de la España decimonónica este colegio rompía con los esquemas y estereotipos sobre la educación de los deficientes. Pionero en la atención de los anormales se hallaba distribuido en dos sesiones, una para niños débiles y otra para niños atrasados, con dos clases cada una que se ocupaban respectivamente de "la educación, enseñanza y tratamiento curativo de los niños débiles y jóvenes atrasados en su desarrollo físico e intelectual hasta el idiotismo" (CEREZO, 2003, p. 124).

\footnotetext{
${ }^{5}$ Ley de Instrucción Pública de 9 de septiembre de 1857. Sección Segunda. Título 1º, capítulo $1^{\circ}$. Artículo 108. "Promoverá asimismo el Gobierno las enseñanzas para los sordo-mudos y ciegos, procurando que haya por lo menos una Escuela de esa clase en cada Distrito universitario, y que en las públicas de niños se atienda, en cuanto sea posible, a la educación de aquellos desgraciados".
} 


\section{Acciones legislativas para los discapacitados en el primer tercio del siglo $\mathrm{XX}$}

En el preámbulo del Reglamento del Colegio Nacional de Sordomudos y Ciegos (1902) el conde de Romanones, a la sazón ministro de Instrucción Pública, incidía en no haber podido diseñar un plan más completo y prestar más atención a los deficientes. La administración española reconocía la desatención y las insuficiencias educativas, pero no adoptó medidas ni resolvió hasta 1914 con la reforma del Patronato de Anormales. Más tarde, Francisco Pereira fundó en Madrid una escuela sanatorio conocida como Instituto Psiquiátrico Pedagógico (1907) para niños con retraso mental y posteriormente una "Sociedad para el estudio y protección de los niños mentalmente anormales y de su educación especial" (1911). En 1911 se creó la sección de anormales en la "Escola de cecs, sordsmuts i anormals" a instancias del ayuntamiento de Barcelona, una sección que respondía a la necesidad escolarizadora de estos discapacitados.

El 22 de enero de 1910 se creó el Patronato Nacional de Sordomudos, Ciegos y Anormales, dependientes del Ministerio de Instrucción Pública. En el decreto de reforma se proponía la agrupación de los niños atendiendo a tres categorías: a) débiles y retrasados mentales. b) imbéciles de grado medio. c) defectuosos mentales. El Patronato desgajó la enseñanza de los ciegos de la de sordomudos por el R.D. 2 de octubre de 1915, escindiendo igualmente las dos reglamentaciones. Así, el Colegio Nacional de Ciegos dejó de ser considerado establecimiento de beneficencia y se convirtió en centro oficial de educación sostenido con fondos del Estado (GASCÓN; STORCH, 2004). Desde las esferas oficiales se legisló a favor de la educación, así lo dispuso en el Real Decreto de 17 de julio de 1913. Las iniciativas de diferentes educadores surtieron un efecto propagandístico y el resultado de sus acciones llegaron al ministerio. Desde instancias oficiales comenzaron a llevarse a cabo acciones para la educación y atención de los deficientes. Antonio Barroso Castillo, ministro de Instrucción Pública y Bellas Artes, creó, por Real Decreto de 22 de enero de 1917, el Patronato Nacional de Anormales. El Patronato tenía amplias competencias, desde la organización de las enseñanzas a la difusión de los conocimientos y la tutela social, además del cometido de inspección de las instituciones 
que recogían a anormales. Después de unos años de incidencias, el 25 de agosto de 1917 se organizaron tres patronatos diferentes (Ciegos, Sordomudos y Anormales), aunque integrados en el Instituto Nacional de Sordomudos, Ciegos y Anormales. Posteriormente, el 13 de septiembre de 1924 se volvieron a agrupar en un solo Patronato Nacional de Sordomudos y Ciegos, aduciendo que no cumplían la misión de atender la educación de los más de 300 alumnos que tenían a su cargo.

A pesar de los intentos de regularización, la intervención educativa era escasa y prácticamente se limitaba a las tareas asistenciales (GUERRERO, 2004). Lo común era el encierro y la exclusión educativa, tanto los niños con retrasos importantes como aquellos que poseían una anormalidad más leve no se escolarizaban. Los problemas eran mayores en los núcleos pequeños de población o más alejados y dispersos donde la escolarización de la infancia era precaria. La falta de conciencia social, la carencia de personal docente cualificado, la falta de métodos pedagógicos, de material didáctico y espacios complicó sobremanera la situación (MÍNGUEZ, 2004).

El Museo Pedagógico Nacional fue una institución señera por su repercusión en la renovación educativa, la introducción y la incorporación de los adelantos que se aplicaban en Europa. La modernización educativa española estaba en sintonía con las innovaciones que llegaban de otros países europeos y con la recepción de las aportaciones de los congresos internacionales. La influencia de los movimientos de renovación pedagógica penetraron en España a través de los profesionales que viajaban al extranjero, de la difusión por medio de conferencias y de la traducción de obras de variado contenido psicopedagógico (CEREZO, 2003).

Bajo gobierno republicano el Ministerio de Instrucción Pública modificó el sistema educativo y mejoró notablemente la educación en todos los niveles (PUELLES, 2002). La normativa reflejaba la preocupación por la infancia anormal, tanto en lo referente a la escolarización como a la formación del magisterio (HERRÁIZ, 2000). Al efecto el Plan Profesional de 1931 contempló en el currículum de maestros un curso de especialización de maestros de Párvulos y otro de maestros para niños Anormales. Una vez instaurada la República, el Ministro Marcelino Domingo, por el Decreto del 22 de septiembre de 1931, reorganizó la Escuela Normal de Profesores 
de Sordomudos y de Ciegos, en la que "los maestros-alumnos se limitaban a seguir los cursos normales, mientras la vida interna del Colegio Nacional de Sordomudos y Ciegos quedaba confiada a personas extrañas al Magisterio Nacional, carentes de la obligada preparación pedagógica, que imprimían al colegio fisonomía de asilo y hospicio". Con esta modalidad pretendían implantar un régimen de convivencia entre niños ciegos y maestros a fin de conseguir un ambiente familiar dentro del Colegio. Más tarde por decreto de 19 de septiembre de 1933 se escindió el Colegio Nacional de Sordomudos y Ciegos. Se organizaron en dos colegios, uno para Sordomudos y otro para Ciegos, delimitado sus funciones y facultades de orden pedagógico y cultural.

En relación con los ciegos el Decreto del 6 de abril de 1934 dispuso, nuevamente, la reorganización del Patronato Nacional de Protección de Ciegos. Se le asignó competencias educativas y asistenciales para llevar a cabo las tareas asistenciales así como educativas de los deficientes visuales. Todos estos centros tenían en común el carácter estatal y el ocuparse de una sola discapacidad, además de concentrar la atención social, sanitaria y pedagógica. En la mayoría de los casos se encargaban también de la formación de los profesionales que posteriormente asumían las tareas educativas (HERRÁIZ, 2000). También en 1933 se refundó el Asilo de Inválidos del Trabajo como centro de educación especial con el nombre de Instituto Nacional de Reeducación de Inválidos. Además en este período se intentó realizar una estadística del número de deficientes españoles y en 1934 se creó el Patronato Nacional de Cultura de los Deficientes.

\section{La dictadura y sus esquemas educativos}

Tras la guerra civil y el gobierno dictatorial desaparecieron los profesionales y grupos pedagógicos innovadores. La educación sufrió una involución; se truncaron los avances científicos y pedagógicos experimentados hasta aquellas fechas (PUELLES, 2002). Algunos de los centros se clausuraron y otros subsistieron de forma precaria, rompiendo con la línea de actuación anterior. La educación especial fue un tema subsidiario y apenas tratado en los primeros años del franquismo. Por el Decreto del Gobierno de Burgos, de 13 de diciembre de 1938 sancionado 
por el Jefe del Estado, fue creada la Organización Nacional de Ciegos de España (ONCE) y un año después se reglamentó su constitución. Entre los fines que se le encomendaron figuraba la tutela de la infancia "ciega y desvalida o cuyos padres sean igualmente ciegos indigentes" y la "unificación, perfeccionamiento y encauzamiento de la enseñanza especial del invidente en todos sus grados, haciéndola eficaz". Siguiendo tales condiciones, la ONCE en su intento de cubrir geográficamente el territorio español se ocupó en primer lugar de crear los Colegios de Madrid, Sevilla, Alicante y Pontevedra. Desde 1941 la ONCE se hizo cargo de la gestión del Instituto Nacional de Ciegos, que de hecho ya la realizaba desde 1928. Con posterioridad, en 1944 desde instancias superiores igualmente se encomendó $\mathrm{ONCE}^{7}$ la Enseñanza Primaria oficial de los no videntes.

La Ley de Enseñanza Primaria de 1945 recogía en su artículo 33 las disposiciones relativas a los centros para la enseñanza elemental de los deficientes mentales ${ }^{8}$. No obstante, fueron los padres los que impulsaron la fundación de centros y sus acciones las que propulsaron la educación especial. Dos años después establecieron las normas concretas sobre las dotaciones tanto económicas como del personal del Colegio Nacional de Sordomudos y la Escuela Nacional de Anormales, así como el Colegio Nacional de Ciegos. A partir de 1947 se fueron diferenciando las respectivas

\footnotetext{
${ }^{6}$ Reglamento de la ONCE, 28 de octubre de 1939.

${ }^{7}$ El Consejo de Estado de 12 de mayo de 1944 encomienda a la ONCE la Enseñanza Primaria oficial de los no videntes "con lo que no sólo se reconoce su experiencia, eficacia y celo puesto en su labor, sino que se utiliza el medio de acción, evitando duplicidad de servicios, que sólo gastos innecesarios, pérdidas de tiempo y de esfuerzos pueden acarrear, sin que por ello pierda el Estado la facultad de crear en el futuro escuelas estatales de esta clase, si cualquier eventualidad lo hiciera aconsejable". Declarando extinguido el Colegio Nacional de Ciegos ordena la desaparición de su plantilla en el Ministerio de Educación Nacional.

${ }^{8}$ Ley de 17 de julio de 1945 sobre Educación Primaria (BOE 18 de julio). Capítulo III.- Escuelas Especiales. Artículo 33. Escuelas de anormales, sordomudos y ciegos."El Estado para atender a la niñez desvalida y proporcionarle educación adecuada, establecerá Escuelas especiales para niños anormales y deficientes mentales y fomentará la iniciativa privada. Asimismo creará y fomentará Escuelas, igualmente especiales, para niños sordomudos, ciegos y deficientes físicos. Todas se regirán por reglamentos peculiares".
} 
tareas de los Colegios Nacionales y la Escuela Central de Anormales, cada uno delimitaba su campo de acción. Años más tarde, en 1963, el Colegio Nacional de Ciegos fue absorbido por la ONCE.

Además de las vagas disposiciones, hasta avanzado el siglo XX no se prestó atención a los niños con deficiencias mentales. Dependiendo del grado de afectación era la actitud y trato hacia ellos, las deficiencias mentales ligeras no se detectaban mientras las deficiencias más graves suponían el encierro en el hogar, en los manicomios o centros asistenciales. Realmente hasta que no se expandió la escolaridad y se detectaron problemas mentales y sensoriales en la heterogeneidad de los menores no se cuestionó la educación especial. La evolución fue lenta, hubo de transcurrir varias décadas para que se incrementara el censo de centros. Realmente se notaron pocos avances entre 1936 y 1955; fueron dos décadas de escasa planificación de estas enseñanzas. En 1953 se creó el Patronato de la Infancia Anormal y se ocupaba de la educación en conexión con los servicios de la Gobernación y Justicia ${ }^{9}$. Este centro se transformó tres años más tarde en Patronato Nacional de Educación Especial (1956), debido al impulso efectuado por la Sociedad Española de Pedagogía. A partir de esta entidad se organizaron las Secciones Provinciales de Educación Especial que funcionaban dentro de los Consejos Provinciales de Educación Nacional. Su función se extendió a la clasificación, elaboración de estadísticas y control de centros a nivel provincial para servir de soporte a la elaboración del Plan Nacional de Educación Especial. No obstante, hubo que esperar hasta la década de 1960 para que se abrieran un mayor número de colegios. La mayoría de estos centros fueron impulsados por la iniciativa privada, sobre todo por la acción de los padres. La Escuela Nacional de Anormales se transformó en el Instituto Nacional de Pedagogía Terapéutica en 1960.

En estos años la situación política española comenzó a variar. Lejos del autoritarismo de décadas anteriores, se apreciaba el efecto de la acción del modelo tecnocrático que pretendía la modernización de España. En 1962 el Ministerio de Educación Nacional pasó a llamarse

\footnotetext{
${ }^{9}$ Por orden ministerial había que clasificar y censar a los deficientes atendiendo a cinco categorías: 1)oligofrénicos, 2) psicópatas, 3) deficientes físicos, 4) deficientes de la vista, deficientes del oído y lenguaje, 5) deficientes afectivos y caracteriológicos.
} 
Ministerio de Educación y Ciencia (MEC) producto de las remodelaciones que se habían llevado a cabo en el equipo de gobierno. Los problemas de los discapacitados sólo habían sido tratados por la medicina hasta aquellos momentos y comenzaron a ser objeto de atención desde el campo educativo. Para poder llevar a cabo la intervención pedagógica pertinente era necesario modernizar la acción educativa, introducir una serie de conocimientos y estrategias (metodologías, materiales didácticos y evaluación), poco difundidas en la escolaridad en España. El interés por la escolaridad de los discapacitados se proyectó también en la formación de maestros especialistas y en el modelo pedagógico también se introdujeron algunas modificaciones metodológicas.

\section{La Modalidad de Educación Especial en la Ley General de Educación}

En el declinar de la dictadura la reforma del sistema educativo pretendía la extensión de la escolarización resolviendo las deficiencias detectadas en anteriores disposiciones. Con la universalización de la escolarización se incorporaron a las aulas un contingente de niños que la escuela excluía (ARNAIZ, 2003, 2004). La Ley General de Educación de 1970 era la primera ley de educación española que contemplaba la educación, al menos a nivel teórico, de todos y todas. La adopción legal del término Educación Especial se produjo, precisamente, a partir de la citada ley y se entendía como una modalidad específica, es decir, como un sistema educativo paralelo al de la educación ordinaria, regido por sus propias normas y por un currículo específico distinto al general. Clasificaba a los alumnos y alumnas de Educación Especial en leves y profundos; los primeros para educarse en colegios ordinarios y los segundos en centros especiales. Sin embargo, este hecho llevaba aparejado otra serie de problemas hasta aquel momento desconocidos. En primer lugar el acceso a la escuela de niños "subnormales" implicaba un estudio previo para su diagnóstico así como la práctica educativa o enseñanza específica que debían recibir. En la práctica sólo tenían acceso a este sistema los deficientes menos afectados que no podían seguir el ritmo de la educación ordinaria puesto que la escolarización no era obligatoria. La normativa 
dedicaba el capítulo VII y sus cinco artículos a la Educación especial ${ }^{10}$. El apartado dos del citado artículo hacía referencia a los niños superdotados, a su escolarización en colegios de régimen ordinarios, procurando que una vez alcanzados sus objetivos comunes pudieran aprovechar sus posibilidades y capacidades intelectuales, siguiendo métodos de enseñanza individualizada. La Ley General de Educación preveía asimismo la creación de aulas de educación especial en centros ordinarios para deficientes ligeros, como medida más avanzada representaba el antecedente de un modelo de educación integrado ${ }^{11}$. Considerando la importancia de la preparación de los profesionales encargados de atender al alumnado se ocupó de la formación de profesorado y del personal necesario. La Orden Ministerial de 30 de noviembre de 1970 reguló la formación del profesorado y creó el título de Profesor de Educación Especial. El Ministerio de Educación y Ciencia se interesó por la formación profesional de los deficientes, de manera que incluyó a la Educación Especial dentro del programa de formación profesional con el objetivo de integrar a estos alumnos en la sociedad $^{12}$. Contempló igualmente las ayudas económicas para los escolares

${ }^{10} \mathrm{El}$ artículo $1^{\circ}$ del Capítulo VII, de la LGE, decía: "Preparar mediante un tratamiento educativo adecuado, a todos los deficientes e inadaptados para una incorporación a la vida social, tan plena como sea posible en cada caso, según sus condiciones y resultado del sistema educativo; y a un sistema de trabajo en todos los casos posibles, que les permita servirse a sí mismo y sentirse útiles a la sociedad".

${ }^{11}$ El artículo 51 de la LGE señalaba que "la educación de los deficientes e inadaptados" se impartiría en centros especiales "cuando la profundidad de las anomalías que padezcan lo hagan absolutamente necesario". Sin embargo, no se aplicó de forma estricta la normativa, porque en los primeros años no se crearon aulas de educación especial. De manera que, entre 1973 y 1975 se impulsaron los centros de educación especial y no la dotación de unidades de educación especial en los centros educativos ordinarios.

${ }_{12}$ El MEC incluyó a la Educación Especial dentro de la Dirección General de Formación Profesional y Extensión Educativa. Dicha Dirección General estaba organizada en tres Subdirecciones Generales, una de ellas se rotulaba Subdirección General de Educación Permanente y Especial. Sus competencias, además de cumplir con la legislación vigente, eran: "La promoción y supervisión de las actividades de Educación Especial, la cooperación con los demás departamentos competentes en la materia y la organización de los servicios del Ministerio de Educación y Ciencia con competencias en Educación Especial". 
que cursaban algún tipo de enseñanzas, según la Orden ministerial de 3 de marzo de 1972, ya que la LGE no regulaba esta cuestión ${ }^{13}$. El MEC a través de los docentes, de los especialistas en Pedagogía Terapéutica junto a los servicios de orientación escolar y los servicios médico-escolares se encargaba de diagnosticar a los alumnos necesitados de Educación Especial ${ }^{14}$. La reestructuración administrativa de este nivel educativo no fue fácil, se tropezó con muchas dificultades para poder aplicar las disposiciones oficiales, por ello fue necesaria la creación de un organismo autónomo que tuviera la misión exclusiva de la progresiva extensión de la Educación Especial. En el año 1973 se constituyó la primera Asociación de Padres de Hijos No Videntes, con el propósito de propulsar la educación de sus hijos, alternativa de la educación integrada en España. Para que este proyecto se pudiese llevar a cabo, la Asociación creó el Centro de Orientación Familiar del Invidente (COFI). Su principal objetivo era trabajar por la integración del no vidente con respecto al resto del colectivo social en general y dentro del núcleo familiar en particular. El COFI fue reconocido por el MEC en

Más tarde la Subdirección de Educación Permanente y Especial se dividió en tres Gabinetes y una Secretaría General. Uno de los Gabinetes se dedicó a la Educación Especial, actuando como órgano de gestión de la Educación Especial.

${ }^{13}$ Según la citada orden se podrían beneficiar de la ayuda "los escolares con deficiencias o inadaptaciones de cualquier tipo que exijan un tratamiento educativo distinto del que se imparte en los centros docentes de régimen ordinario" del grupo de edades comprendidas entre los 3 y 18 años, y 21 para los de formación profesional; siempre y cuando su anomalía no les obligara a "ser atendidos preferentemente en Instituciones de carácter predominantemente clínico o asistencial”. Poco después, la Orden de 30 de junio de 1972 ampliaba el tipo de ayudas (transporte, residencia, comedor, asistencia técnica, rehabilitación y atenciones complementarias).

Entre los años 1971 y 1975 se adoptaron medidas importantes para la Educación Especial, apoyadas por el Plan de Desarrollo Económico y Social (1972), entre ellas la transición de los "minusválidos" y "subnormales" a la actividad laboral, considerando la importancia de la formación especial "con el fin de obtener la máxima incorporación a la sociedad de los españoles afectados".

${ }^{14}$ Entre los años 1971 y 1975 se adoptaron medidas importantes para la Educación Especial, apoyadas por el Plan de Desarrollo Económico y Social (1972), entre ellas la transición de los "minusválidos" y "subnormales" a la actividad laboral, considerando la importancia de la formación especial "con el fin de obtener la máxima incorporación a la sociedad de los españoles afectados". 
septiembre de 1982, fue nombrado en el mismo año Primer Grupo de Apoyo por el INEE (Instituto Nacional de Educación Especial) y el 24 de febrero de 1983 fue también designado por la Junta de Gobierno del Real Patronato de Educación y Atención a Deficientes como experiencia piloto para la integración social de los niños con deficiencias visuales.

En 1975 se creó el Instituto Nacional de Educación Especial (INEE), organismo autónomo dependiente del Ministerio de Educación, pero se hallaba escasamente planificado. Comenzó a funcionar cinco años más tarde de la promulgación de la LGE, y, tres años después, en 1978 se elaboró el primer plan estatal sobre la materia. Las iniciativas de los padres creando asociaciones suplieron la falta de acción de la administración pública. Así en 1976 se creó el Real Patronato de Educación Especial que servía de nexo entre la iniciativa privada y la pública, impulsaba y coordinaba todas las acciones educativas. Posteriormente su actividad se concretó en el Plan Nacional de Educación Especial (1978) proyectando los principios y criterios resultó un hecho relevante en el sentido de que con la defensa del colectivo se adelantaba a la mentalidad de la época y sirvió de base para la Ley de Integración de Minusválidos aprobada en $1982^{15}$. Además, la Constitución de 1978 en su articulado recogía y especificaba la amplia gama de derechos de los disminuidos ${ }^{16}$. En el marco constitucional quedaba fijada "la igualdad de derechos y libertades de los minusválidos antes la Ley, como todos los ciudadanos".

El siguiente referente legislativo de importancia que encontramos fue la Ley 13/1982 de Integración Social del Minusválido, en la que

\footnotetext{
${ }^{15}$ Ley de 7 de abril de 1982. Se trataba de una ley que recogía las declaraciones de derechos internacionales de Naciones Unidas (deficientes mentales de 1971 y de minusválidos de 1975) así como los derechos de la Constitución española aprobados en 1978. El minusválido se definía como: "Toda persona cuyas posibilidades de integración educativa, laboral o social, se hallen disminuidas como consecuencia de una deficiencia, previsiblemente permanente, de carácter congénito no, en sus capacidades físicas, psíquicas o sensoriales".

${ }^{16}$ En el artículo 49 recoge: "Los poderes públicos realizarán una política de previsión, tratamiento, rehabilitación e integración de los disminuidos físicos, sensoriales y psíquicos, a los que prestarán la atención especializada que requieran y los ampararán especialmente para el disfrute de los derechos que este Título otorga a todos los ciudadanos".
} 
se establecían los principios básicos de atención a las personas con discapacidad en todos los ámbitos, entre ellos el educativo: normalización, sectorización e integración. El Real Decreto de 15 de octubre de 1982, de Ordenación de la Educación Especial, sintetizó los aspectos básicos sobre los que debía regirse la educación especial. La falta de coherencia entre la teoría y la práctica condujo a la promulgación de la Orden Ministerial de 20 de marzo de 1985, con la finalidad de hacer efectiva la integración en los centros de Enseñanza General Básica (EGB). Para evitar la marginación dentro del sistema educativo, el MEC a través de la Subdirección General de Educación Especial estableció un conjunto de medidas recogidas en el Real Decreto de Ordenación de la Educación Especial de 6 de marzo de $1985^{17}$. El citado Real Decreto se constituyó en el referente para la organización de la Educación Especial en España. Los proyectos curriculares y las programaciones de aula tenían que responder a las necesidades de todos los alumnos, con el objetivo de que todo el alumnado pudiera participar en la vida de los centros. Posteriormente el Real Decreto 334/1985 de Ordenación de la Educación Especial marcó otro hito al establecer que el currículum de Educación Especial había de basarse en todo caso en el ordinario, si bien teniendo en cuenta las diferencias individuales. Este decreto señaló el comienzo experimental del programa de integración ${ }^{18}$ en España y como consecuencia inmediata se creó en 1986 el Centro Nacional de Recursos para la Educación Especial, dependiente del Ministerio de Educación.

A partir de la Ley General de Educación se conformó un nuevo tipo de profesor: el maestro de pedagogía terapéutica. La consolidación de la formación en las instituciones encargadas de la formación de maestros, así como el tipo de formación que éstas propiciaban estuvo delimitada por la forma en que se relacionaron varios procesos como el papel de las

${ }_{17}$ El Real Decreto 334/1985 de Ordenación de la Educación Especial define la Educación Especial "como una parte integrante del sistema educativo y la concreta como el conjunto de apoyos y adaptaciones para que estos alumnos y alumnas puedan hacer realmente efectivo su derecho a la educación".

${ }^{18}$ El programa de integración tuvo una duración de 8 años, 3 de ellos con carácter experimental y 5 de expansión de la integración de alumnos con necesidades educativas especiales a centros ordinarios. 
nuevas enseñanzas, el crecimiento, la diversificación y la estratificación del sistema educativo, así como la apropiación de nuevas teorías y métodos pedagógicos. Los docentes tutores de los alumnos escolarizados en Educación Especial eran denominados Maestros de Pedagogía Terapéutica, intervenían tanto en centros específicos de educación especial o como profesores de apoyo a la integración en centros ordinarios.

Las directrices legislativas de la LGE no resolvieron las diferencias ni respondieron a las necesidades del alumnado, porque atender de modo especial a los diferentes y clasificarlos en grupos reproducía la exclusión. La escuela es un espacio de socialización y aprendizaje y debe propiciar la interacción educativa desde la diferencia.

\section{La escolarización y la atención a la diversidad}

La práctica de la LGE de integrar alumnos con necesidades educativas especiales en "aulas ordinarias" (DÍAZ, 2003, p. 191) era el reflejo de reconocer una situación evidente pero no asumida. Dicha integración supuso la introducción de una metodología adecuada en la planificación diaria del trabajo en el aula al tiempo que la atención temprana de las diferencias individuales. No se logró porque no se erradicaron las prácticas y actitudes discriminatorias hacia el alumnado. Se siguió cuestionando sus capacidades y enjuiciando de forma anticipada los resultados con prejuicios negativos. Esta situación contribuyó a que no cambiara el panorama hacia la integración de los alumnos en el currículum general (AINSCOW, 1995, 1999).

En los últimos años se han abierto otras perspectivas fortalecidas con nuevos conceptos como "la diversidad" y "la escuela inclusiva" (DÍAZ, 2003:192), con el objetivo de cambiar la escuela para que tuvieran cabida todos y todas, superando antiguas creencias basadas en la desigualdad y la discapacidad $^{19}$. La Ley General de Educación no garantizaba la educación para todos, mantenía activas las desigualdades, incluso con una respuesta

\footnotetext{
${ }^{19}$ Herraiz afirma que "la educación debe ser inclusiva, integradora e individual, con lo que el concepto de Educación Especial se diluye y no presenta diferencias con el concepto de Educación" (Herraiz, 2000, p. 31).
} 
curricular también diferente. Encorsetaba de acuerdo con las capacidades intelectuales, excluyendo y segregando alumnos y alumnas considerados especiales. Atender de modo especial a los diferentes y clasificarlos en grupos reproducía la exclusión.

El nuevo marco normativo ${ }^{20}$ emanado de la LOGSE propició cambios sustanciales (ARNAIZ, 2003, 2004; VLACHOU, 1999). El nuevo enfoque superaba al modelo tradicional de homogeneidad, evidenciaba los términos de educación especial y discapacidad para situarse en la diversidad. Con la aprobación de la LOGSE se respetaban las diferencias, se contemplaba la diversidad con el objeto de corregir la discriminación y las desigualdades (Título V, art. 63.2). La LOGSE pretende compensar las desigualdades ${ }^{21}$, desde un marco flexible ofrece respuestas al alumnado con necesidades educativas especiales, con la finalidad de conseguir una adecuada adaptación que le permita el máximo desarrollo personal y social. El alumnado con discapacidad adquiría un reconocimiento legislativo, que le amparaba en condiciones igualitarias. A partir de la década de los años noventa del pasado siglo se refiere más a "la inclusión" que a la integración de los alumnos que precisan de una atención especial (GENTO, 2007, p. 581). El término tiene mayor alcance y supera la integración, porque además de abarcar los aspectos curriculares y pedagógicos incorpora los de carácter social y físico. Incluye a todos los alumnos sin excepción, porque asisten a la escuela para aprender y estar con los compañeros de edad, formando parte plenamente de la comunidad educativa. Para lograr esa igualdad, al margen de las diferencias de capacidad intelectual, física, sexo, origen y clase social se precisa de "una educación de calidad"(GENTO,

\footnotetext{
${ }^{20}$ Según el Real Decreto 696/1995 se propondrá la escolarización en los centros de educación especial "a los alumnos con necesidades educativas especiales asociadas a discapacidad psíquica, sensorial o motora, graves trastornos del desarrollo o múltiples deficiencias que requieran a lo largo de su escolarización adaptaciones curriculares significativas en prácticamente todas las áreas del currículo, o la provisión de medios personales y materiales poco comunes en los centros ordinarios y cuando se prevea, además, que en estos centros su adaptación e integración social sería reducida".

${ }^{21}$ El Preámbulo de la ley recoge: "La educación permite avanzar en la lucha contra la discriminación y la desigualdad, sean estas por razón de nacimiento, raza, sexo, religión u opinión, tengan un origen familiar o social, se arrastren tradicionalmente o aparezcan continuamente con la dinámica de la sociedad".
} 
2007, p. 581), con programas y recursos adaptados al tratamiento inclusivo de la diversidad ${ }^{22}$. La segregación escolar desde la escuela induce también a la segregación social y a la marginación de la vida activa. Sin embargo, la inclusión fomenta actitudes positivas y favorece la integración en la vida adulta. Por ello es necesario comprender que todos somos diferentes y que denominaciones como discapacidad y desigualdad no resuelven nada. "No basta con integrar a los alumnos en centros y aulas ordinarias" (DIAZ, 2003, p. 192), es fundamental que los alumnos se sientan integrados, incluidos en la vida del aula, del centro y de la comunidad. Porque la inclusión es para todos los alumnos y miembros de la comunidad, por ello ha quedado superado por la integración, va más allá, cambia la actitud y la concepción, pero además diferencias de capacidad, destrezas, cultura, género, etnia...

Una escuela que atienda a la diversidad debe entenderla como un elemento que favorece la educación de calidad para todos, sin excepciones, puesto que ya no es necesario basarse en categorías para proporcionar una educación adecuada. Porque, al ser diferente ya no es algo negativo dentro de una sociedad plural y de una escuela abierta a todos, es un derecho que tiene todo ser humano, y como derecho no se puede vulnerar (DÍAZ, 2003, p. 193).

Resulta más apropiado el empleo del término diversidad que educación especial, y se relaciona con un currículum igual para todos. Se trata de un currículo más abierto, flexible y participativo acorde con las particularidades de cada estudiante. En este ámbito se considerada la diversidad como un rico recurso de apoyo al aprendizaje y a la enseñanza (ALEGRE, 2000). La constatación de diferencias individuales en una sociedad plural debía favorecer la diversidad, de manera que las antiguas prácticas educativas quedaban desfasadas. Para evitar los descalificativos y clasificaciones tradicionales, se usó el término necesidades educativas especiales y, posteriormente, evolucionó al de diversidad. Las personas con discapacidad, ya sean físicas o psíquicas, tienen un espacio de

22 "La calidad de la educación debe incluir la defensa de la equidad o igualdad de derecho de todas las personas, incluidas las que muestran alguna acusada diversidad, a beneficiarse de la misma calidad de la educación" (Gento, 2007, p. 581). 
reconocimiento dentro de la diversidad. La atención a la diversidad es un término amplio que engloba múltiples aspectos que precisan ser considerados en la educación (ALEGRE, 2000). En la realidad educativa están presentes las diversidades físicas e intelectuales, las diversidades étnicas, religiosas, ideológicas, lingüísticas, género, culturales, económicas, etc. Toda una serie de diferencias que marcan ritmos distintos de aprendizajes, de habilidades, de experiencias, de rendimientos y de desarrollo de talentos (DÍAZ, 2003). En el marco escolar la diversidad se manifiesta a nivel individual, social, cultural y a nivel de factores relacionados con deficiencias físicas, psíquicas y sensoriales. Este último grupo "está constituido por aquellos alumnos que tradicionalmente han sido segregados del sistema educativo ordinario" (DÍAZ, 2003, p. 195). Un colectivo ampliamente despreciado y que ha sufrido distintas formas de discriminación, desde el rechazo escolar a la marginación.

\section{Hacia la escuela inclusiva}

La exclusión se ha mantenido en distintas formas y diferentes grados en el sistema educativo, de ahí que uno de sus objetivos y retos haya sido detectar y eliminar sus causas. Ya hemos señalado que el planteamiento de la educación especial ha evolucionando y, dada su complejidad, han ido surgiendo cantidad de debates y discusiones encaminadas hacia la integración y, más recientemente, a la inclusión. Las formulaciones más tradicionales de la inclusión han estado asociadas con el ámbito de las necesidades educativas especiales o discapacidades. Curiosamente aquéllos que han ingresado al sistema educativo regular desde instituciones especiales suelen mantenerse relativamente aislados del resto de sus compañeros, y se les trata con diferencia tachándoles como "alumnos de integración o inclusión" en lugar de ser tratados con "normalidad" (AINSCOW, 2004). Porque la inclusión significa insertar al alumno en un espacio de socialización y aprendizaje propiciando la interacción educativa y su participación en el proceso de construcción del conocimiento: Desde la diferencia propiciar el aprendizaje (AINSCOW, 2004). Mel Ainscow incide en mejorar las condiciones de enseñanza aprendizaje y por ello enfatiza en que el gran reto del sistema educativo es 
“eliminar los procesos de exclusión" en razón de la capacidad, al género, a la religión, a la etnia y a la clase social (AINSCOW, 2005, a). Plantea la utilización de experiencias como recursos para superar los obstáculos de aprendizaje de los alumnos presentes en el modelo tradicional, y defiende la investigación participativa porque el aprendizaje que se construye contribuye al desarrollo del pensamiento (AINSCOW, 2002). Por ello se deben emprender nuevas experiencias para desarrollar un sistema de inclusión más extenso. Porque la inclusión educativa requiere de un programa amplio que aborde las diferentes esferas sociales, culturales y económicas, para lograr la interacción escuela -sociedad (AINSCOW, 2004). En palabras de García

La escuela inclusiva es aquella que ofrece oportunidades educativas a todos los estudiantes, y las ayudas curriculares, personales y materiales que son precisas para su progreso académico y personal. Esta perspectiva de la inclusión no debe reducirse a una simple cuestión curricular, organizativa y metodológica, sino que ha de buscar una manera distinta de entender la educación, que debe trascender a la vida en sociedad e implica una nueva filosofía de valores. Contribuye a reducir la discapacidad, pues permite mantener las expectativas de los estudiantes ofreciendo modelos de comportamiento y aprendizaje adaptados, y enriquece al grupo al diversificar su composición interna (GARCÍA, 2008, p. 95).

Debe atender a la comunidad educativa en sentido amplio y a la formación del profesorado, a la organización de grupos heterogéneos de alumnado en las aulas, a la adaptación del currículum, la dinamización del aprendizaje e interrelación de los alumnos, al intercambio de experiencias, así como la desaparición de aulas separadas y la participación de los padres en la tarea conjunta, la dotación económica suficiente y la eliminación de la competitividad (GENTO, 2007). Las escuelas eficaces son escuelas educativamente inclusivas y están dispuesta a ofrecer nuevas oportunidades a aquellos alumnos que han presentado dificultades. Se interesa por los alumnos, identifica a aquéllos que tienen problemas para seguir el ritmo marcado o se sienten desplazados de los objetivos (AINSCOW, 2003). 
Pero "El éxito de la inclusión depende del éxito inclusivo de los centros (...) depende de la formación y del modo en que se utilizan los recursos (...) que no es tarea de unos pocos, sino de muchos, de todos" (CARDONA; CHINER, 2006, p. 300-301). No obstante el cambio ha sido más teórico, a pesar del cambio de terminología y evitarse las clasificaciones y catalogaciones, se aprecian muchas contradicciones y continúa organizando al alumnado como grupo homogéneo. Para lograr con éxito la educación inclusiva o educar en el respeto a las diferencias es preciso prestar atención a la formación del profesorado, al cambio de actitudes y a la metodología de la enseñanza como uno de los principales retos para atender a la diversidad de población escolar (DÍAZ, 2003). Sin embargo, una de las limitaciones ha sido la formación del profesorado y las dificultades que estos tienen para captar sus propias necesidades y carencias de formación, así como la efectividad para la adaptación del currículum (CARDONA; CHINER, 2006). El profesorado es la piedra angular en este proceso pero su preparación no es suficiente para afrontar los retos que plantea la escuela inclusiva y para afrontar con éxito la formación de los alumnos. Motivo por el cual se precisan actuaciones docentes que promuevan y apoyen el aprendizaje organizando clases heterogéneas que fomenten la cooperación, el diálogo y el respeto. Además, al margen de la formación continua, es necesario superar el desfase entre la formación inicial de los docentes y la realidad educativa, las universidades deben replantear los perfiles y adaptarlos a la praxis y situaciones cotidianas. En la actualidad la escuela inclusiva tiene que responder a diversidad de necesidades, intereses y características. Debe ser un lugar flexible y adaptado que acoja a todos sus miembros independientemente de sus individualidades. La inclusión lleva aparejada una serie de exigencias y modificaciones de conductas, de actitudes y creencias del conjunto de la sociedad.

\section{Conclusiones}

Hemos realizado un breve recorrido histórico de la Educación Especial en España, siguiendo las etapas fundamentales. En la evolución repasamos los aspectos más importantes que reflejan el tratamiento de los deficientes. Esa mirada retrospectiva nos sitúa en el plano social de las 
creencias y prejuicios, en la formación de etiquetas hacia determinadas personas y grupos en función de su discapacidad y, desde la óptica educativa, en la marginalidad y las insuficiencias. El alumnado con discapacidad y con dificultades de aprendizaje sufría situaciones discriminatorias en todos los ámbitos. Es preciso remontarse hasta 1857 para encontrar la Educación Especial incorporada al sistema educativo, aunque sólo fuera para atender a ciegos y sordos. La transición entre el discurso oficial y la práctica no ha sido fácil. Las pretensiones del legislador con los discapacitados no siempre contaron con los medios suficientes para aplicarlos a la realidad. Otra controversia representó la forma de identificar y clasificar a los niños y niñas con dificultades, pues se perdía en clasificaciones ambiguas y dispersas que complicaba la identificación entre los propios profesionales (médicos y educadores). De manera que resultaba imposible la sintonía profesional y la actuación conjunta en la atención de la infancia con necesidades educativas. Más bien se originó un conflicto entre la Pedagogía y la Medicina al no clarificarse las competencias de unos y otros. Pero los conocimientos psicopedagógicos fueron cobrando protagonismo, convirtiéndose en saberes que contribuyeron al progreso en la práctica educativa. Desde las instituciones se intentaba perseguir los objetivos trazados desde el currículum prescrito, pero no siempre se alcanzaban. Fueron muchos los condicionantes, desde la integración física en los centros, por la marginación que existía de hecho al ser considerados "bobos" o "subnormales" por utilizar uno de los calificativos más genéricos, a la falta de espacio y materiales didácticos.

La Ley General de Educación pese a las limitaciones representó un avance importante para la Educación Especial y al respecto constituye un referente básico. Desde el reconocimiento como modalidad educativa ha propulsado su transformación. En el marco de la ley y la práctica educativa se observó la necesidad de diferenciar la enseñanza y el currículum para atender las necesidades de todo el alumnado. Una de las dificultades para su extensión fue la escasa preparación del personal docente, debido a que la formación de los profesionales, la cualificación y actualización de los docentes no siempre fue la deseada, porque la inversión efectuada no fue proporcional a las necesidades de preparación. Sin embargo, los maestros y los estudiantes también incidieron en estos procesos y, al 
hacerlo, construyeron una nueva cultura escolar. La formación inicial de los docentes no se desprende únicamente de los planes y programas de estudio, de las teorías pedagógicas que los inspiran, o de las políticas educativas impulsadas por las autoridades. La formación en sentido amplio está presente en la cultura escolar, en la disposición de los espacios y del tiempo, en las reglas de convivencia formales e informales, en las rutinas, los ritos, los mitos, los saberes, las formas de reclutamiento y las promesas de la escuela, en las prácticas y los discursos que se construyen día a día.

Las comunidades autónomas han regulado estas enseñanzas especiales a partir de la descentralización del estado español y la transferencia de las competencias educativas a las diversas comunidades autónomas. Pero todas las transferencias no se llevaron a cabo al mismo tiempo y no hubo auténtica sintonía en el ritmo de aplicación en la integración escolar. Un desfase que en ausencia de normativa propia se ha regido por lo dispuesto en el ámbito estatal por los Reales Decretos 696/1995 y 299/1996. Los citados decretos han evolucionado de acuerdo con la atención prestada por las respectivas comunidades autónomas a la Educación Especial. Con frecuencia, cuando no se ha elaborado ningún protocolo y carecen de un desarrollo normativo propio, estas enseñanzas se regulan por el marco general trazado por el MEC, asumiendo los diseños curriculares específicos. Después de los logros alcanzados con la LGE y las pertinentes modificaciones, la LOGSE ha llevado a cabo un tratamiento específico, ha realizado las adaptaciones curriculares y ha acotado al alumnado con necesidades educativas especiales. Para garantizar la igualdad de oportunidades tiende a eliminar la integración porque su objetivo es la integración completa o inclusión; el currículo es abierto y flexible, con la posibilidad de adaptación al alumnado y a la realidad del centro. En Estados Unidos estos planteamientos se iniciaron en la década de 1980 y posteriormente se extendieron por otros países, como Canadá y Europa. En España han llegado con cierto retraso; los tópicos comenzaron a cambiar a la luz de la transformación curricular planteada con la LOGSE.

Desde la historia del pensamiento pedagógico la Educación Especial ha marchado estrechamente unida a las representaciones sociales, a los estereotipos con carácter de exclusión y en su evolución se ha ido posicionando en el reconocimiento a la igualdad. Desde la educación 
se han ido deconstruyendo esas prácticas discriminatorias, trabajando por los derechos y la dignidad de todas las personas. Su reconocimiento pasa por la descatalogación y el desaprender los prejuicios. Porque el deficiente tiene derecho a la diferencia y a no ser excluido, a asumir sus características singulares y la sociedad debe contribuir a que pueda vivir con su deficiencia.

La escuela debe cambiar y la sociedad construir nuevos valores para normalizar la diferencia, pues la errónea interpretación de la integración ha implicado su negación y es preciso potenciar las capacidades de cada alumno tal y como es, aceptar las diferencias y fomentar las cualidades y singularidades de cada uno, para que puedan integrarse en la sociedad plural del siglo XXI. Apostar por una escuela inclusiva lleva consigo superar los viejos tópicos y romper con los estereotipos que generan discriminación, porque su modelo integrador contribuye a respetar las diferencias y los derechos de los otros. Sin embargo, el rostro social de los discapacitados continúa siendo invisible y silenciado. Las barreras y prejuicios hacia un colectivo que en España se acerca a los cuatro millones son inmensas, hecho que entorpece el proceso de inclusión social de acuerdo con sus características. Sirva de ejemplo el caso de unas $32 \mathrm{mil}$ personas aproximadamente que con síndrome de Down reivindican un espacio propio y un rol activo en la sociedad.

\section{Referências}

AINSCOW, M. (1995). Necesidades especiales en el aula. Madrid: Narcea, AINSCOW, M. (1999). Desarrollo de una escuela para todos. Revista: Infancia y aprendizaje, n. 85:33-58.

AINSCOW, M. (2002) Rutas para el desarrollo de prácticas inclusivas en los sistemas educativos. Revista: Educación, n. 327:69-82

AINSCOW, M. (2003). El desarrollo de las escuelas inclusivas. En El fracaso escolar: Una perspectiva internacional pp. 319-329.

AINSCOW, M.; ALONSO, P.; DURÁN, D.; CHEITA, G.; FONT, J.; MARIN, N.; MIQUEL, E.; PARRILLA, Ma A.; RODRÍGUEZ, P. (2004). Educar sin excluir. Cuadernos de Pedagogía. No 331:50-53. 
AINSCOW, M. (2005, a-). La mejora de la escuela inclusiva. Cuadernos de Pedagogía, $\mathrm{n}^{\mathrm{o}}$ 349:78-83.

AINSCOW, M. (2005, b-). Para comprender el desarrollo del sistema educativo inclusivo. Electronic journal of research in educational psychology, Vol 3-7: 5-20.

ALEGRE DE LA ROSA, O. (2000): Diversidad Humana y Educacion. Málaga: Aljibe.

ARNAIZ, P. (2003). Educación inclusiva: una escuela para todos. Málaga: Aljibe.

ARNAIZ, P . (2004). La educación inclusiva: Dilemas y desafíos. Revista: Educación, Desarrollo y Diversidad, Vol. 7(2): 25-40.

CARDONA, CARDONA, M Y CHINER, E. (2006) Uso y efectividad de las adaptaciones instructivas en aulas inclusivas: Un estudio de las percepciones y necesidades formativas del profesorado, Bordón, 58(3): 287-303.

CEREZO (2003). Los inicios de la psicopedagogía en España. En G. OSSENBACH (Coord.). Psicología y Pedagogía en la primera mitad del siglo $X X$. Madrid: UNED.

DÍAZ, E. M. (2003) Atención a la diversidad: condiciones de la escuela para todos, Bordón, 55(2):191-203.

ESPAÑA, J.A. (2002). El sistema Braille. Málaga: Consejería de Educación/Junta de Andalucía.

GASCÓN, A. y STORCH, J.G. (2006). Fray Pedro Ponce de León, el mito mediático. Los mitos antiguos sobre la educación de los sordos. Madrid: Editorial Universitaria Ramón Areces.

GASCÓN, A. y STORCH, J.G. (2004) Historia de la Educación de sordos en España. Madrid: Editorial Universitaria Ramón Areces.

GARCÍA, P. C. (1993). Una escuela común para niños diferentes: la integración escolar. Barcelona: PPU.

GENTO, S. (2007) Requisitos para una inclusión de calidad en el tratamiento educativo de la diversidad, Bordón 59(4) 581-595. 
GUTIÉRREZ, I. (1997). Introducción a la historia de la logopedia. Madrid: Narcea.

GUERRERO, J. F. (2004) "Los seguidores de Osiris, la turbulenta historia de la educación especial” en: GUERRERO LÓPEZ, J. F. y PÉREZ GALÁN, R. (Coords.). La pizarra mágica: una visión diferente de la historia de la educación. Málaga:Aljibe, pp. 99-116.

HERRÁIZ, M. (2000) La organización de la enseñanza de los niños anormales en los inicios de la educación especial española (1900-1936), Bordón, 52 (1): 31-38.

MINGUEZ, C. (2004). "Evolución del encierro de personas discapacitadas o historia de la institucionalización en educación especial" en GUERRERO LÓPEZ, J.F. y PÉREZ GALÁN, R. (Coords.) La pizarra mágica: una visión diferente de la historia de la educación, Málaga: Aljibe, pp. 131-156. PUELLES, M. (2002). Educación e ideología en la España contemporánea. Madrid: Tecnos.

PUELLES, M. (2005). Educación, Igualdad y diversidad cultural. Madrid, Biblioteca Nueva.

VLACHOU, A. D. (1999). Caminos hacia una educación inclusiva. Madrid: La Muralla.

VICENTE, A. y VICENTE, M. P. (2001). Una aproximación a la Historia de la Educación Especial, Murcia, Diego Marín. 